\title{
Capítulo X \\ DE SÍSIFO A PIGMALIÓN: CÁTEDRAS JURÍDICAS Y CURRÍCULO EN LA USTA
}

Jhon Fredy Maldonado Ruiz ${ }^{1}$

\section{Introducción}

El currículo es la concreción de todas las apuestas de una institución por lograr la finalidad de la educación. Ello implica una coherencia entre la realidad y los procesos de enseñanza y de aprendizaje. Lo cual se ha de concretar en el ejercicio diario de las prácticas docentes.

La Universidad Santo Tomás comprende que una forma de realizar la concreción de su Filosofía Institucional se da por medio del Departamento de Humanidades y Formación Integral (DHFI), el cual se constituye en un proceso transversal que, desde lo académico, se relaciona con las diferentes unidades, departamentos y facultades ${ }^{2}$.

1 Licenciado en Filosofía, Ética y Valores Humanos. Especialista en Educación con énfasis en Evaluación Educativa. Magíster en Educación.

2 Departamento de Humanidades y Formación Integral, Informe de autoevaluación con fines de la acreditación institucional. Documentos Marco Departamento de Humanidades y Formación Integral. (Bogotá: Universidad Santo Tomás, 2014), 4. 
Por esa línea, una de las labores del DHFI es acompañar el ejercicio académico de las facultades por medio de su estrategia de la ruta formativa, en la cual se establecen los componentes obligatorio $^{3}$ y flexible ${ }^{4}$. En este caso se acompaña a la Facultad de Derecho mediante las denominadas cátedras jurídicas, las cuales se abren como espacios para el diálogo interdisciplinar entre las ciencias jurídicas y las problemáticas de la realidad.

De lo anterior se suscita la necesidad de comprender las apuestas del currículo en la Universidad Santo Tomás, concretadas por medio del Departamento de Humanidades y Formación Integral, a través de su ruta formativa en las cátedras jurídicas. En otras palabras, acercarse a las perspectivas desde las que el Departamento está pensando y concretando la educación, el currículo, la evaluación.

En razón a lo anterior se propone una analogía entre las narraciones de Sísifo y Pigmalión y el ejercicio educativo en la Universidad Santo Tomás, más propiamente del Departamento y cátedras ya mencionadas, acompañadas en la Facultad de Derecho. Desde allí se presentarán las concepciones de educación, currículo y evaluación que se cristalizan en el ejercicio docenteestudiante. En consecuencia, en el apartado de dos narraciones como referentes, Sísifo y Pigmalión, se presentará el sentido en el que se comprenden las historias para su utilización como analogía del actuar docente en las cátedras jurídicas; luego en Pigmalión, currículo y educación, se trata de enmarcar la discusión desde la comprensión de los conceptos de currículo y educación; seguido a ello en Sísifo, relación currículo y cultura, se menciona la íntima relación que existe entre la cultura y las apuestas curriculares de una institución; posteriormente con Sísifo, el evaluador, y Pigmalión, que aprende a valorar, se construye la relación y diferencia entre la evaluación como un mecanismo meramente artificial y descontextualizado y como articulación del proceso educativo a la realidad de la persona para la toma de decisiones y el aprendizaje; en Pigmalión, la ruptura con la criatura, se llega al planteo que, dentro de la educación, el ejercicio del maestro se da para que el estudiante logre su estado de perfección, apartándose de su creador para llegar a ser gestor de su conocimiento y proyecto de vida.

3 Este componente hace referencia a las cátedras de Filosofía Institucional, antropología, epistemología, cultura teológica, filosofía política y ética, en ellas el estudiante va relacionando el conocimiento disciplinar con los saberes propios de las cátedras que estructuran e interrelacionan saberes desde una cosmovisión que asuma la realidad: mundo, sociedad, historia, Dios, hombre como totalidad integrada y como propuesta de vida. Política Curricular (Bogotá: Universidad Santo Tomás, 2004b), 33 - 35.

4 Son cátedras enmarcadas en la personalidad de figuras modélicas de la Orden Dominicana, en las cuales se realiza profundización del conocimiento y de los saberes en relación a las problemáticas actuales. Ibíd., 35 - 37. 


\section{Dos narraciones como referentes: Sísifo y Pigmalión}

Las historias de Sísifo y Pigmalión comparten una característica en común, son "vidas" que se transformaron en referentes para las vidas de otros. Estas narraciones son icónicas en el sentido en que sirven a otros como analogías para comprender y asumir posturas frente a determinadas realidades morales, éticas, actitudinales, epistemológicas, etc.

Albert Camus y Philippe Meirieu, como otros tantos autores, se sirven de estas historias para referir situaciones concretas del pensamiento y de la realidad humana. Camus retoma el mito diciendo que los dioses habían condenado a Sísifo a subir sin cesar una roca hasta la cima de una montaña desde donde la piedra volvía a caer por su propio peso. Habían pensado, con algún fundamento, que no hay castigo más terrible que el trabajo inútil y sin esperanza 5 .

El autor comenta esto como el castigo más efluente para quien despreciaba la muerte y amaba los placeres de la vida. Además, lo utiliza como una imagen hermosa para demostrar lo ineficaz de un trabajo sin sentido, rutinario y lejano a todo conocimiento de ello. Esto último lo más oprobio, toda vez que no permite levantar la cabeza sino, por el contrario, ata a las cadenas y a seguir en la sombras de la caverna, sin conciencia.

Por otro lado Meirieu, utilizando las palabras de Ovidio, trae a la mente a Pigmalión quien:

Es un escultor taciturno, quizá incluso algo misántropo, que vive solo y consagra toda su energía a la elaboración de una estatua de marfil que representa a una mujer tan hermosa "que no podía deber su belleza a la naturaleza". Una vez terminada su obra, Pigmalión se comporta con su estatua de un modo extrańo: "la besa e imagina que sus besos le son devueltos", le pone las mejores ropas, la colma de regalos y de joyas, y por las noches se acuesta junta a ella ${ }^{6}$.

La historia no para allí, comenta el autor que Venus saliendo de unas fiestas, en honor suyo, pasó y, admirada por aquella situación, accedió a las solicitudes del escultor enamorado de su obra: le dio vida para que se convirtiera en la mujer "perfecta" de su creador.

Estas dos historias van a servir para mostrar la labor docente, en ocasiones sin sentido, alienada y desprovista de conciencia frente a lo que se hace; en otras oportunidades dolorosa por cuanto se toma al estudiante, se le va moldeando a través de una historia de amor y desamor, de entusiasmo y desaliento por ver la obra constituida en un ser independiente, autónomo y que ya no permite seguir siendo influenciado. El creador entiende que la finalidad de su obra es

5 Albert Camus, El mito de Sisifo (3a Edición ed.), (Madrid: Alianza Editorial S.A., A. 1985), 59.

6 Philippe Meirieu, Frankenstein educador (Barcelona: Laertes, 1998), 31-32. 
apartarse de su creador. Finalmente dos historias que muestran lo relevante de comprender la apuesta curricular institucional de la USTA en la práctica docente en las cátedras jurídicas.

\section{Pigmalión, currículo y educación}

Jurjo Torres hace referencia a la importancia que tiene el ser conscientes de la finalidad de la educación a la hora de poner en práctica cualquier mecanismo pedagógico. Pues ello permite vislumbrar y orientar las prácticas y apuesta que se realizan en la escuela ${ }^{7}$. Si se reconoce el sentido de la educación desde la perspectiva de la institución es factible que las acciones construyan puentes entre lo que se piensa y lo que se hace. De esta manera Torres refiere que:

La concreción de las finalidades de la escolarización es el eje principal que nos permite revisar de manera continuada los distintos planteamientos de la función y actividad docente. [...] Una meta de esta categoría (preparar a los jóvenes para ser ciudadanos) requiere, por consiguiente, que la selección de los contenidos del curriculum, los recursos y las experiencias de enseńanza y aprendizaje que día a día caracterizan la vida en las aulas, las formas de evaluación y los modelos organizativos promuevan la construcción de los conocimientos, destrezas, actitudes, valores, normas, etc., precisos para ser un buen ciudadano y ciudadana ${ }^{8}$.

De lo anterior se pueden destacar tres elementos constitutivos del currículo: finalidad, perspectiva y praxis. Donde la finalidad se comprende como el componente teleológico que permite evidenciar cuál es la actividad misional de la institución en relación con el de la educación en general; la perspectiva hace referencia al lugar o postura epistemológica desde la cual se asume la concepción de esa finalidad y que de una u otra manera ha de ser comunicada y asumida por la comunidad en que se desarrolla; por último, la praxis da sentido al resto de componentes, toda vez que es la ejecución de la finalidad y desde la perspectiva en un medio real, en un contexto determinado, y que ha de plantear un ejercicio de transformación.

7 Aquí el concepto de "escuela" hace referencia a toda etapa de educación formal, desde la primaria hasta la posgradual.

8 Jurjo Torres, Globalización e interdisciplinariedad: el currículo integrado (4a ed.). (Madrid: Ediciones Morata, 2006), 131. 
El currículo será, entonces, la apuesta de una institución a la hora de concretar los ejercicios educativo (como sistema), pedagógico (como orientación teórica) y didácticos (como apuesta hacia el aprendizaje). En esa medida, de la coherencia entre finalidad, perspectiva y praxis deviene en la pertinencia del currículo. En el caso de la Universidad Santo Tomás, desde su objetivo misional se busca que las personas, actores de la educación, asuman una postura frente a su realidad, de manera que respondan a las exigencias de la vida humana desde lo ético, creativo y crítico?.

Por lo anterior, se entiende que la Universidad Santo Tomás establezca la educación como un mecanismo que le permite llevar a la persona hasta su estado superior en cuanto persona ${ }^{10}$, y a esto le llama promover: es necesaria una relación de igualdad, horizontal cooperativa, entre el docente y el estudiante ${ }^{11}$. Este último es quien ulteriormente se convierte en el principal agente de su proceso de formación. El maestro, según Tomás de Aquino, acompaña, ayuda a salir, economiza, alimenta mediante símbolos y señales que el estudiante toma para formar su propio conocimiento $^{12}$.

Ahora bien, en lo anterior se ve implícitamente una aproximación a lo que la Universidad Santo Tomás comprende como persona. Ser perfectible es su potencialidad, a la manera de la virtud aristotélica, su fuerza para perfeccionarse. De ahí que se afirme la dignidad humana -fundada en la semejanza con Dios-, la centralidad de la existencia humana, la necesidad del desarrollo de todas sus posibilidades ${ }^{13}$. Y a este desarrollo de todas sus posibilidades le llama formación integral. En otras palabras, el objetivo substancial de la USTA es el de llevar gradualmente al hombre hasta un lugar más elevado de su existencia como persona, o lo que es lo mismo "ascenso hasta el 'estado perfecto de hombre' [...] hasta alcanzar una capacidad estimativa autónoma y responsabilidad habitual en el uso de la libertad, guiada por la 'prudencia' o aptitud para la acción valiosa, de cara a los distintos desafíos situacionales"14.

9 Universidad Santo Tomás. Proyecto Educativo Institucional PEI. (Bogotá: Universidad Santo Tomás, 2004a)

10 Ibíd., 17.

11 Ibíd., 26.

12 Diego Gracia Ortiz, Guillermo de Ockham, O.F.M. El nominalismo y su irrupción en la Universidad de París (Bogotá: Siglo del Hombre y Universidad San Buenaventura, 2010), 31.

13 Universidad Santo Tomás, Proyecto Educativo Institucional PEI, Op. Cit., 22.

14 Ibíd., 23-24. 
En esa medida, el hombre será un ser racional, capaz de autodirigir su propia vida e intervenir como agente de convivencia en medio de su contexto. Un ser que se hace responsable de sus acciones en medio de otras personas, pues son estas las que le permiten la consolidación y enrutamiento hacia su fin último, la felicidad.

En consecuencia, no se busca un ejercicio de reproducción, sino de transformación; no se concibe la educación como una continuidad de las perspectivas y prácticas a la manera de Sísifo, rutinaria y alienada; por el contrario, se espera que sea un trabajo como el de Pigmalión, que al final no pueda contener a su criatura, pues ella posee autonomía. Se crea para liberar.

En otras palabras, como plantea Torres acerca del currículo con el cual se pretende "además de desarrollar capacidades para la toma de decisiones, facilitar a los estudiantes y al propio profesorado una reconstrucción reflexiva y crítica de la realidad, tomando como punto de partida las teorías, conceptos, procedimientos, costumbres, etc., que existen en esa comunidad y a las que se debe facilitar el acceso" ${ }^{15}$.

Estar inmersos en su realidad, asumiendo una postura determinada, impulsa a las personas a desarrollar o fortalecer capacidades que les permitan comprender de manera crítica su lugar en la realidad. En esa medida, la educación le ha de proporcionar, desde las diferentes perspectivas epistemológicas, teóricas y métodos, las herramientas para acceder a lo que sucede en su comunidad, de suerte que pueda ser agente de transformación. Ello le lleva a desprenderse de su creador, de su maestro, para adoptar sus propias apuestas frente a su contexto.

\section{Sísifo, relación currículo y cultura}

Como se dijo anteriormente, para que exista pertinencia, el currículo ha de estar en directa consonancia con las necesidades, prácticas, perspectivas y finalidades que orientan a la comunidad en un contexto determinado. Con ello se ha de tener cuidado, porque se puede caer en el ejercicio de Sísifo, seguir la misma rutina diariamente, lo que en palabras de Marx sucede con el capitalismo, el fenómeno de la reproducción: "Cada proceso social de producción es, al mismo tiempo, un proceso de reproducción [...] La producción capitalista además [...], produce no solo

15 Torres, Op. Cit., 132. 
comodidades, no solo plusvalía, sino también produce y reproduce la relación capitalista, en un lado el capitalista, en el otro el trabajador asalariado" 16 .

Para el caso, el aula se puede transformar en un lugar donde se dé la reproducción de los esquemas de la realidad, puesto que "la enseñanza y el aprendizaje que tienen lugar en las aulas representan una de las maneras de construir significados, reforzar y conformar intereses sociales, formas de poder, de experiencia, siempre con un significado cultural y político" ${ }^{17}$.

En palabras de Henry Giroux, las escuelas funcionan como agencias de la reproducción social y cultural, esto es, legitiman la racionalidad capitalista y sostienen las prácticas sociales dominantes. La escuela puede fungir como refuerzo o como transformación de la realidad, como legitimadora de los esquemas hegemónicos o como desarticuladora de estereotipos. Esto se puede evidenciar cuando en las escuelas la teoría se da por un lado y la práctica por otro; cuando la escuela no aborda las necesidades, problemas, lenguajes y apuestas de la realidad en que vive el estudiante.

Se da un vacío de contexto en el discurso de la escuela. En palabras de Torres, el currículo queda al margen de las voces ausentes. Las cuales son constituidas por aquellas "culturas o las voces de los grupos sociales minoritarios y/o marginados, que no disponen de estructuras importantes de poder (que se) acostumbran a ser silenciadas, cuando no estereotipadas y deformadas para anular sus posibilidades de reacción" 18 .

Voces que ante el currículo no siempre existen, y al no hacerse presentes quedan personajes (con sus vidas, procesos, experiencias, etc.) sin referenciar en la escuela, lo cual equivale a abandonar todo intento de pertinencia, equidad e justicia de cualquier proceso educativo.

Lo anterior lleva a perder la posibilidad de proporcionar dentro de los discursos de la escuela lo que la persona ve en la realidad. Es decir, no se le permitirá contrastar las teorías articuladas por el currículo en función de su contexto. Alejándose de la concepción del currículo como una construcción cultural que busca asegurar la integración de las personas a las necesidades y particularidades de la vida. Pues como apunta Guillermo Londońo:

En muy diversos sentidos el estudio del currículo es también el estudio de la lectura, se enfatiza en la idea de que este sentido histórico y cultural trasciende las propuestas de formación a partir de la racionalidad técnica, y que por los proyectos de formación no deben ser ajenos a las

16 Karl Marx, Capital, I. (Moscow: Progress Publishers, 1996), 531-532.

17 Torres, Op. Cit., 137.

18 Torres, Op. Cit., 133. 
exigencias de desarrollo y transformación cultural correspondientes con los contextos sociales e históricos. [...] Las apuestas educativas, máxime en el nivel superior, no pueden ser ajenas ni a las realidades socio-históricas de los agentes educativos, ni a la necesidad de participar como agentes de transformación social ${ }^{19}$.

Y en palabras de Shirley Grundy:

El curriculum no es un concepto, sino una construcción cultural...Una forma de organizar un conjunto de prácticas educativas humanas...Una perspectiva cultural de curriculum se refiere a las experiencias de las personas consiguientes a la existencia del curriculum, y no a los diversos aspectos que lo configuran...Eso significa que hemos de buscar el curriculum, no en la estantería del profesor, sino en las acciones de las personas inmersas en la educación ${ }^{20}$.

De allí la necesidad de comprender la acción social del maestro, quien se constituye, desde sus perspectivas teórico-epistemológicas ${ }^{21}$, en agente de transformación o consolidación de las apuestas culturales y sociales.

El currículo, entonces, tiene que contribuir a situar a los estudiantes (la mujer, el hombre, el homosexual, gay, lesbiana, negro, indígena, blanco, profesor, estudiante, entre otros) en el mundo, lo que implica, entre otras cosas, redescubrir su historia, recuperar la voz perdida ${ }^{22}$. O como refieren Grinberg, S. y Levy, E., citados por Guillermo Londoño:

Si la escuela no está exenta de las luchas de poder, sino que es campo de fuerza, de constitución de sujetos, de producción de realidades, entonces, lo que se trata es de la necesidad de desarrollar prácticas pedagógicas que hagan algo más que leer de un tirón las ideologías particulares tal y como se producen dentro de textos particulares; se trata de desarrollar metodologías que ilustren

19 Guillermo Londoño Orozco, Pertinencia del currículo en el ámbito universitario, en Currículo y prácticas pedagógicas: voces y miradas con sentido crítico, eds, Cristhian James Díaz Mesa y Dirléia Fanfa Sarmento, (Bogotá: Universidad de la Salle, 2011), 109-110.

20 Shirley Grundy, Producto o praxis del curriculum. (Madrid: Morata S.L., 1998),19 - 21.

21 María Paz Sandín Esteban, Investigación cualitativa en educación. Fundamentos y tradiciones. (Barcelona: McGraw-Hill/Interamericana de España, S. A. U., 2003).

22 Torres, Op. Cit., 142. 
cómo a partir de instituciones particulares, economías de poder y lugares de lucha, surgen y se legitiman representaciones particulares ${ }^{23}$.

En consecuencia, se puede decir que el DHFI oferta para los estudiantes de Derecho cátedras como las de Bartolomé de las Casas: identidades culturales y justicia social, Francisco de Vitoria: derechos humanos; las cuales se consideran espacios para el diálogo entre lo disciplinar y diversas corrientes filosófico-epistemológicas, de manera que el estudiante tenga la oportunidad de comprender, a partir de núcleos problémicos que a la vez se actualizan conforme a problemáticas que emergen permanentemente de la realidad, las diversas perspectivas en que se realizan los procesos de construcción identitaria, la incidencia de las políticas y escuelas filosóficas en dichos procesos, la apuesta por los derechos humanos como apuesta por el respeto del otro y el papel del profesional tomasino como agente de construcción de culturas más equitativas, incluyentes y justas.

Esto último implica que para establecer vasos comunicantes entre la realidad, el derecho y las diferentes cátedras de la ruta formativa, se haga necesario establecer, dentro de las prácticas docentes, una perspectiva justa de la evaluación, la cual el DHFI comprende, en la óptica de la Universidad Santo Tomás, no como un mero ejercicio de la memoria, sino como un proceso que permite tanto a docentes como a estudiantes reconstruir los procesos de enseńanza y de aprendizaje para tomar decisiones.

\section{Sísifo el evaluador}

La actividad de evaluar ha sido mediada por la perspectiva del evaluador. Qué entiende por conocimiento, estudiante, enseñanza, aprendizaje, educación y ser humano; además, por los condicionamientos que ofrecen las vicisitudes del contexto sociocultural en medio del cual se desarrolla: instruir para la sociedad industrial, formar para la vida pública, educar para la paz, promover para la emancipación y la autonomía. Y, en esa medida, este proceso toma matices e identidades que responden a la realidad y que terminan perfilando a la persona evaluada. Los evaluados terminan siendo identificados, valorados, seleccionados, escogidos de acuerdo al "ojo-observador".

23 Londoño, Op. Cit., 132. 
Así, Tomás Escudero hará referencia a las actividades generadas en la china imperial para seleccionar funcionarios, también a la manera de escoger en los primeros siglos de la era cristiana los pasajes bíblicos, los aportes de los filósofos y teólogos. De la misma manera, comentará la entrada formal de la evaluación en las universidades medievales. Prácticas todas estas que, en un principio, se establecerán para diferenciar y seleccionar a estudiantes o material a enseñar; lo cual se constituía en un proceso que no necesariamente estaba enmarcado por referentes teóricos explícitos ${ }^{24}$.

Posteriormente, con la entrada en escena de los diferentes movimientos pregoneros de la educación, de la civilización, de la libertad, de la igualdad, de la fraternidad, de la sociedad industrial, que buscaban la emancipación del hombre sobre la naturaleza, se hará cada vez más importante la formación y cualificación para desempeñar alguna labor dentro de la sociedad, y por ese camino será relevante comprobar que efectivamente las personas cumplan con los requisitos que se le exigen.

Con la modernidad se hará explícito el apoyo en la observación, la experimentación, los datos y los hechos como fuentes del conocimiento verdadero. Lo cual va a generar un amplio despliegue de creatividad en el desarrollo de mecanismos que permitan confirmar, revisar, medir. El objetivo fundamental será detectar y establecer diferencias individuales dentro del modelo del rasgo y atributo que caracterizaba las elaboraciones psicológicas de la época.

Congruente con la perspectiva de aquel momento, lo importante será establecer las medias, las variables, las frecuencias y demás datos que les permitirán describir, controlar y predecir los fenómenos dentro del campo educativo. Se trasladó, así, el positivismo de las ciencias naturales o fácticas al campo de las perspectivas sociales o humanas.

Es evidente que estos grandes desarrollos se dan al margen de los evaluados e, incluso, de los evaluadores. Será una carrera por la comprobación de los datos, teorías e hipótesis planteadas. De esta manera, igual que Sísifo, los desarrolladores de tests e instrumentos evaluativos dejarán de lado realidades fundamentales en la comprensión del mismo sentido de evaluar, para concentrarse en el objeto de su deseo, en la piedra que habría de subir por la pendiente, sin caer en cuenta de que, al día siguiente, esta se encontraría en el mismo lugar de donde la tomaron. En otras palabras, se preocuparon más porque la persona o las realidades evaluadas encuadraran en la herramienta [objetivos] (esto hacía que se validaran sus hipótesis) que por tratar de explicar

24 Tomás Escudero Escorza, Desde los tests hasta la investigación evaluativa actual. Un siglo, el XX, de intenso desarrollo de la evaluación en educación. Revista Electrónica de Investigación y Evaluación Educativa - RELIEVE, Vol. $9 \mathrm{~N}^{\circ} 1$ (2003): 12. 
lo que realmente sucedía en el fenómeno, perdieron la comprensión de por qué evaluaban, el sentido de ello, la finalidad. Lo importante era la herramienta, la piedra.

\section{Pigmalión aprende a valorar}

El desarrollo tecnológico y de los medios de comunicación traerá consigo nuevas formas de ver la realidad. Las distancias se acortan gracias a las líneas telefónicas, a los móviles satelitales y la aeronáutica. Se pueden referir lugares jamás visitados físicamente fruto de la maravillosa pantalla grande, los ordenadores permiten realizar cálculos antes nunca vistos $\mathrm{y}$, además, organizar cantidad de información en espacios reducidos. Esto lleva a que las relaciones con los otros y con lo otro se transforme y, por ende, la manera de posicionarse frente a la realidad.

Todos estos cambios exigirán nuevas formas de revisar los procesos de educación. Situación que tendrá que involucrar, como las redes de intercomunicación, a todos los agentes y fenómenos del contexto. Muestra de ello será la proliferación de concepciones y metodologías que buscan abordar el qué es, el qué y cómo ha de abordarse y conducirse en el proceso evaluativo ${ }^{25}$. Se traslada, como en las ciencias sociales, el valor hacia la persona evaluada en medio de y para un contexto real.

Se comprende, de esa manera, a la evaluación como el acto consistente en emitir un juicio de valor a partir de un conjunto de informaciones sobre la evolución o los resultados de un estudiante, con el fin de tomar una decisión ${ }^{26}$. Esto implicará que la evaluación tiene que tomarse como una práctica cotidiana (¿acaso no lo es?) realizada por todos y que necesariamente afecta como medio para desarrollar y potencializar desde y para las subjetividades ${ }^{27}$.

De allí que se piense la evaluación como formativa, pues "la razón de ser de la evaluación es servir a la acción; acción educativa debe entenderse desde el punto de vista formativo, que como profesor le debe (pre)ocupar antes de cualquier otra consideración”28.

25 Escudero, Op. Cit., 21.

26 Universidad Santo Tomás, Proyecto Educativo Institucional PEI, Op. Cit., 21.

27 Universidad Santo Tomás, Modelo Pedagógico, Op. Cit., 35-36.

28 Universidad Santo Tomás, Proyecto Educativo Institucional PEI, Op. Cit., 25. 
La evaluación, por ende, tiene como finalidad propiciar el espacio para que tanto el estudiante como el profesor adquieran, mediante ella, conocimientos que le permitan orientar su ejercicio hacia nuevas metas o procesos. Les ha de dar la posibilidad de advertir las dificultades que se puedan experimentar, de suerte que se busquen nuevas estrategias educativas más exitosas.

A la manera de Pigmalión, que buscaba formar una obra perfecta, la juzga, la ve perfecta, situación por la cual termina cobrando vida y, por lo tanto, autonomía de su creador. Esto es lo que hoy en día se ve en los intentos por evaluar, que se van haciendo complejos, más contextualizados, dependientes de las necesidades, sabiendo que es un proceso sociopolítico, colaborativo, de enseñanza y aprendizaje, continuo, recursivo, altamente divergente. En definitiva, es un proceso que crea realidades, vidas e identidades en otros.

Esta idea es entendida en medio de las cátedras jurídicas, pues permiten al docente construir puentes entre la realidad, las ciencias jurídicas y los discursos de las cátedras del DHFI. Además, la valoración entendida de tal manera se constituirá en un fuerte recurso para concretar la formación integral promovida por la USTA. Esto se ve reflejado cuando el estudiante se asume parte de un todo, no como alguien aislado, que en su comprensión de sí mismo abarca y se pregunta por el otro.

\section{Pigmalión, la ruptura con la creatura}

Para la USTA, como para Santo Tomás de Aquino, dentro del concepto de persona se pueden considerar tres aspectos: que es educable, perfectible y unión indivisible de cuerpo y espíritu. Esta situación lleva necesariamente al objetivo misional, "promover la formación integral"29, la promotio e conductio ${ }^{30}$, conducir hasta un estado de perfección. $Y$ desde esta optimista concepción de persona, que posee el docente del DHFI, se configura la idea de que la educación busca el desarrollo y fortalecimiento de las potencialidades en el estudiante, de suerte que camine hacia su "perfección como persona". Se piensa la educación como un proceso intencional, por el cual el

29 Ibíd., 63.

30 Universidad Santo Tomás, Modelo Pedagógico, Op. Cit., 22-23. 
ser humano se desarrolla orientándose siempre hacia la mayor perfección de su propio ser ${ }^{31}$, esto es, hasta su estado de virtud.

$Y$ es en ese sentido que los conceptos de integralidad y perfección se constituyes en quicios fundamentales para la educación en la USTA, pues indica que necesariamente el estudiante tendrá la constante posibilidad de irse perfeccionando en todas sus dimensiones como persona ${ }^{32}$. Lo cual implica "haber integrado ocho hábitos operativos del 'hombre en cuanto hombre': aptitud para asumir la realidad: desarrollo de la inteligencia, cultivo de la ciencia, conquista de la sabiduría (comprensión de totalidad); aptitud para obrar moralmente: prudencia, justicia, fortaleza y templanza; y 'arte', aptitud para hacer, crear, producir"33.

Esto implicará apartarse, romper con su docente. Ir caminando hacia un punto donde haya adquirido, dentro de sus potencialidades, fuerzas, habilidades, destrezas y competencias, el poder de ser, tener y saber el conocimiento por y para sí mismo. Por ello será necesario que aunque se desee una obra para sí, como en el caso de Pigmalión, se cree para y desde los otros, desde y para la cultura. De aquí que en las cátedras jurídicas, comprendiendo que su finalidad es relacionar los conocimientos de las ciencias jurídicas con las problemáticas de la realidad cambiante, se dé privilegio a las dimensiones del comprender (ámbito del conocer), el obrar (ámbito de la acción valiosa), el hacer (ámbito de la acción transformadora y productiva), el comunicar (ámbito de la acción comunitaria mediante los distintos lenguajes) ${ }^{34}$.

Entonces, igual que en la historia de Pigmalión, es claro que el "efecto expectativa", puede afectar los resultados de los proceso de concreción curricular en medio de las prácticas pedagógicas docentes. Esta narración da, además, otro aspecto de la misión educativa y es el rompimiento con la obra. Se espera fabricar hombres y mujeres para la cultura, no personas pasivas, sino autónomas, con vida propia. Es allí donde radica lo desconcertante, se busca que la obra desborde los límites del creador para que desde la libertad y autonomía acoja a su maestro; en la realidad aunque se desee, esto no siempre tiene el final feliz. La obra escapa, o sencillamente no toma vida ${ }^{35}$.

31 Ibíd., 23.

32 Ibíd., 24; $27-28$.

33 Universidad Santo Tomás, Proyecto Educativo Institucional PEI, Op. Cit., 67.

34 Ibíd., 77.

35 Meirieu, Op. Cit., 34 - 35. 


\section{Conclusión}

Es conveniente cerrar diciendo que dentro de las cátedras jurídicas se pretende superar la visión mecanicista de Sísifo sobre el ejercicio docente como una actividad rutinaria y sin sentido. Donde se ve al estudiante como un dato más, desvinculado de la realidad y formado para cumplir con unas labores en la sociedad. Que adopta las cátedras como espacios independientes y cerrados en sus propios discursos, desvinculados con la realidad de la persona y su contexto.

Superación que, desde la óptica optimista de Santo Tomás de Aquino, implica la comprensión de la educación como promoción de una persona hasta su estado de virtud, de autonomía. Situación que se evidencia, en las cátedras jurídica, cuando se le da la posibilidad al estudiante de preguntarse, desde diversas perspectivas epistemológicas, por su realidad, de manera que logre unir los discursos de las ciencias jurídicas y filosóficas para la solución de problemas de una manera ética, creativa y crítica, abarcando sus múltiples dimensiones como persona, para, en determinado momento, tener el poder de apartarse de su creador, como en la historia de Pigmalión, para cultivar un saber integral sobre la condición humana y su destino, con miras a iluminar el porvenir de la humanidad ${ }^{36}$.

\section{Referencias}

Bernal, Aurora. La educación del carácter. Pamplona: Eunsa, 1998

Botero, Juan José. Rawls contra Rawls. Bogotá: Universidad Nacional, 2005.

Camus, Albert. El mito de Sisifo (3a Edición ed.). Madrid: Alianza Editorial, S.A., 1985.

Departamento de Humanidades y Formación Integral. Informe de autoevaluación con fines de la acreditación institucional. Documentos Marco Departamento de Humanidades y Formación Integral. Bogotá: Universidad Santo Tomás, 2014.

Escudero Escorza, Tomás. Desde los tests hasta la investigación evaluativa actual. Un siglo, el XX, de inteso desarrollo de la evaluación en educación. Revista Electrónica de Investigación y Evaluación EducativaRELIEVE, Vol $9 \mathrm{~N}^{\circ} 1$ (2003): 11 - 43.

Giroux, Henry. Teorías de la reproducción y la resistencia en la nueva sociología de la educación: un análisis crítico. Bogotá: Universidad Pedagógica Nacional, 1983). http://goo.gl/HzMMxe

36 Universidad Santo Tomás, Proyecto Educativo Institucional PEI, Op. Cit., 67. 
Gracia Ortíz, Diego. Guillermo de Ockham, O.F.M. El nominalismo y su irrupción en la Universidad de París.

Bogotá: Siglo del Hombre y Universidad San Buenaventura, 2010.

Grundy, Shirley. Producto o praxis del curriculum. Madrid: Morata S.L., 1998).

Londoño Orozco, Guillermo. Pertienecia del currículo en el ámbito universitario. En H. J. Díaz Mesa, fsc.,

\& D. Fanfa Sarmento, Currículo y prácticas pedagógicas: voces y miradas con sentido crítico (págs. 105 -

134). Bogotá: Universidad de la Salle, 2011).

Marx, Karl. Capital, I. Moscú: Progress Publishers, 1996).

Meirieu, Philippe. Frankenstein educador. Barcelona: Laertes, 1998.

Quintar, Estela. La enseñanza como puente a la vida. México: Instituto Politécnico Nacional, Instituto Pensamiento y Cultura en América Latina, 2006.

Sandín Esteban, María Paz. Investigación cualitativa en educación. Fundamentos y tradiciones. Barcelona:

Mcgraw-Hill/Interamericana de Espańa, S. A., 2003.

Torres, Jurjo. Globalización e interdisciplinariedad: el currículo integrado (4a ed.). Madrid: Ediciones Morata, S.L., 2006.

Universidad Santo Tomás. Proyecto Educativo Institucional PEI. Bogotá: Universidad Santo Tomás, 2004

Universidad Santo Tomás. Política Curricular. Bogotá: Universidad Santo Tomás, 2004.

Universidad Santo Tomás. Módulo de Evaluación. Licenciatura en Filosofía y Lengua Castellana. Bogotá: Universidad Santo Tomás, USTA, 2009.

Universidad Santo Tomás. Modelo Pedagógico. Bogotá: Universidad Santo Tomás, 2009. 
